\title{
Granulomatous inflammation of dura mater - a rare side effect after application of hemostatic and insulation materials in case of two-stage operation of huge meningioma
}

\author{
Jarosław Andrychowski1 ${ }^{1,2,6}$, Zbigniew Czernicki ${ }^{1,2,6}$, Anna Taraszewska ${ }^{3,6}$, Małgorzata Frontczak-Baniewicz ${ }^{5}$ Ewa Przytuła ${ }^{4,6}$, \\ Marta Zębala ${ }^{6}$ \\ 1Department of Neurosurgery, Medical University of Warsaw, Bielański Hospital, Warsaw, Poland, 2Department of Neurosurgery, \\ Mossakowski Medical Research Centre, Polish Academy of Sciences, Bielanski Hospital, Warsaw, Poland, ${ }^{3}$ Department of Experimental \\ and Clinical Neuropathology, Mossakowski Medical Research Center, Polish Academy of Sciences, Warsaw, Poland, ${ }^{4}$ Department \\ of Pathomorphology, Medical Centre for Postgraduate Education, Bielanski Hospital, Warsaw, Poland, ${ }^{5}$ Electron Microscopy Platform, \\ Mossakowski Medical Research Center, Polish Academy of Sciences, Warsaw, Poland, ${ }^{6}$ Bielański Hospital in memoriam Jerzy \\ Popiełuszko, Warsaw, Poland
}

\begin{abstract}
Haemostatic and isolating materials may cause local reactions as a foreign body. The case presented here of intracranial granulomatous lesion pertains to a patient operated in two stages due to a huge meningioma. During the first operation the tumour was partially removed. Because of persistent intraoperative haemorrhage haemostatic flakes of Oxycel and Spongostan were applied locally. In order to cover the lack of the dura, an insulation material - Tachosil was used. Histological examination of the tumour specimens confirmed the preoperative diagnosis of benign meningioma, mainly of the angiomatous subtype. The second stage of operation was performed after 3 months and the meningioma was completely removed, as well as dura mater and meningioma attachment with its oncological margin. The resected dura mater was thickened and histologically showed intensive granulomatous infiltrations and foreign body reactions most likely to Oxycel. Clinically no local and general infection and improper healing was observed after the first and the second treatment stage, but an allergic skin lesions and increased eosinophils in peripheral blood smear were noted. It was stated that systemic allergic reaction and granulomatous inflammation of dura mater were an uncommon response to the applied haemostatics and/or insulation material used during the first operation. This report show that haemostatic and isolating agents, generally used in neurosurgical procedure, may rarely cause local granulomatous processes considered as delayed hypersensitivity and the foreign body reactions. Therefore, they may hinder morphological assessment of the tissues during reexploration and must be differentiate with the other infectious and non-infectious granulomatous processes.
\end{abstract}

Key words: resorbable hemostatics, hypersensitive granulomatous reaction, foreign-body granuloma, dura inflammation, neurosurgery.

Communicating author:

Jarosław Andrychowski, Department of Neurosurgery, Medical University of Warsaw, Bielański Hospital, ul. Cegłowska 80, 01-809 Warsaw, phone/fax: +48 2256904 90/22 83500 05, e-mail: j.andrychowski@wp.pl 


\section{Introduction}

Insulation and haemostatic materials are widely used in neurosurgery and other surgical specialities. Resorbable substances that locally support haemostasis are produced on the basis of animal proteins (horse, pig) and additionally human proteins, in the form of flakes, fibrin, sponge and other was used. In special situations, e.g. hypersensitivity to the aforesaid proteins, one can observe local or generalized reactions of the organism. The literature describes inflammatory infiltrations of a foreign body type, formation of granulomas or pseudo-tumour regrowth as a rare reaction to haemostatic materials used in surgery $[4,9,11,14,16]$. Quite often postoperative formation of intracranial granulomas, resulting in a mass effect (space occupying lesions), can mimic tumour recurrence $[1,3,8,11,12,15,16]$. Allergic reactions to foreign collagen, used in surgery as a haemostatic agent, are also described $[2,10]$.

We present a case of the immense granulomatous inflammation of dura mater, assessed three months after application of haemostatic and insulation agents in the patient with a two-stage operation of a huge meningioma. Before surgical treatment the patient had been allergic to many factors including animal proteins. The presented case illustrates possible incidence of granulomatous processes as a rare side effect of treatment with haemostatic and insulation materials, widely used in surgery.

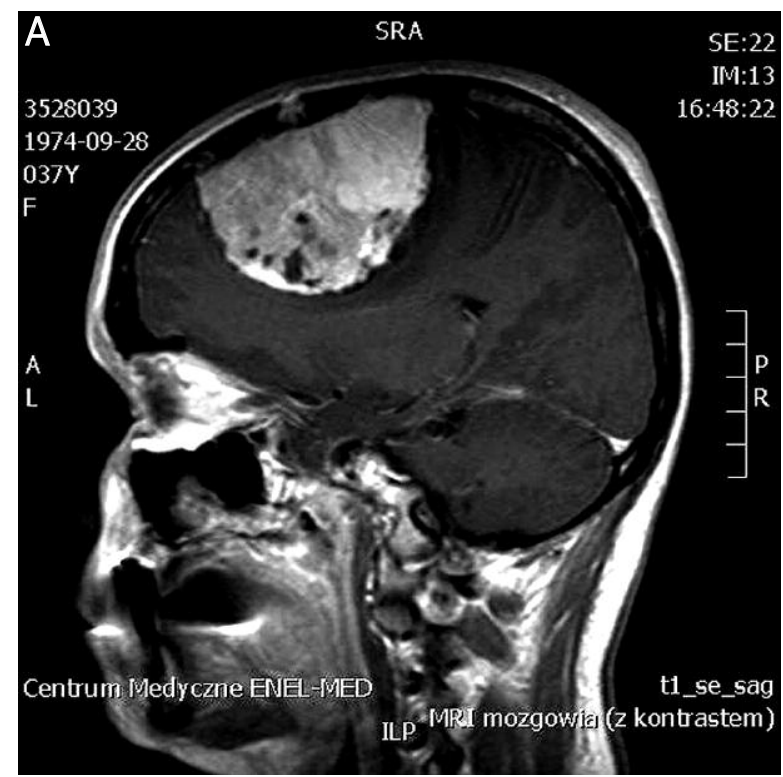

Fig. 1. Intracranial huge meningioma in the left hemisphere before the first operations, the wide attachment in convexity and falx region, mass effect.

\section{Description of the case}

The patient, woman aged 37, was admitted due to a large meningioma located in the left frontoparietal area. The beginning of clinical symptoms were headaches and epileptic seizures. From the case history it was obvious that the patient had already been allergic to many factors presenting in the environment. Preoperative MRI examination in multiple planes revealed a huge tumour located in the left hemisphere with a very significant mass effect. It was the tumour with dural attachment in convexity and falx. The dimensions in the widest planes were: $75 \mathrm{~mm}$ in sagittal, $65 \mathrm{~mm}$ in horizontal and $60 \mathrm{~mm}$ in the vertical plane (Fig. 1A, B). The patient was referred to surgical treatment. The embolization procedure was not performed before due to diversified vasculature sources of the meningioma. Two-stage removal of the tumour was performed, with an interval of three months between operations. During the first stage, the decompressive craniectomy was performed and tumour of a benign meningioma morphology was partially removed with a part of the meningioma's attachment. The decision to finish the first stage of surgery was made due to a significant blood loss. To support haemostatic mechanisms, Oxycel (oxidized cellulose - Oxycel, Becton Dickinson, USA) and Spongostan (gelatin sponge - Ferrosan, MD, Denmark) were used. In order to cover the lack of the dura mater, own pericranium was sewn

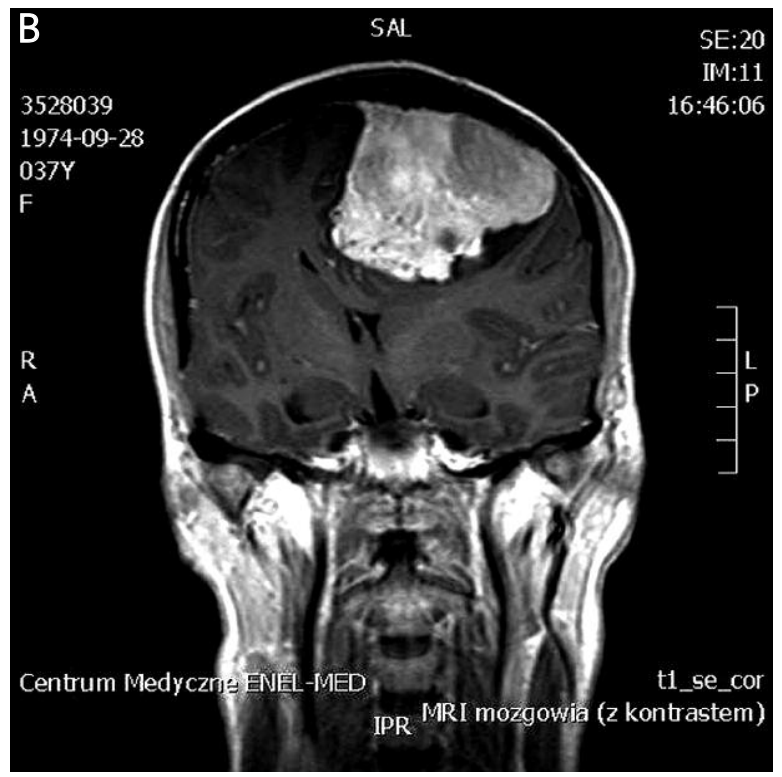
$\mathrm{E}: 20$ 

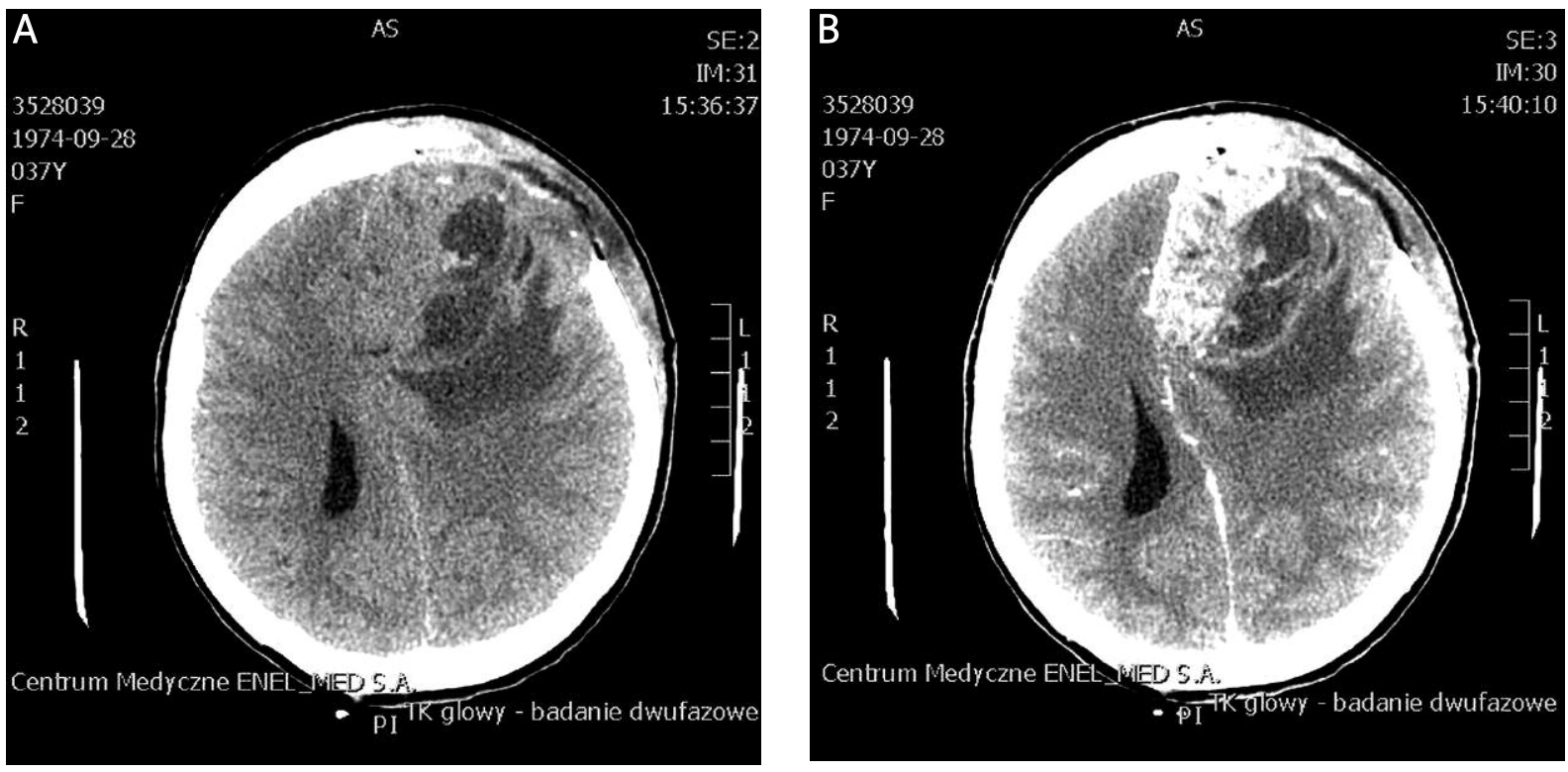

Fig. 2. CT control examination after first stage of operation, visible bone decompression and partial resection of tumour.

in and, additionally, an insulation material - Tachosil (Takeda - Japan) was used. The effect of the first surgery was assessed in the CT-partial resection of the tumour, local mass effect and bone decompression (Fig. 2A, B). The patient left the clinic in a very good condition, without neurological symptoms. Histopathological examination of the tumour showed benign meningioma. The second surgical treatment stage was planned.

During control examination, after approximately 6 weeks, the patient complained of the small allergic reactions - she had skin pruritus, without rash, and insignificant swelling of eyelids and other soft parts of the face. She was treated with anti-allergic drugs and showed improvement. Due to intensification of headaches, the second stage of surgical treatment took place after 3 months. An opening was made in the previous place, any abnormalities were observed in situ after the partial resection of the meningioma. No suppuration or remains of haemostatic materials were observed. The meningioma was completely removed - Simpson 0. Dura mater around the tumour was resected with oncologic margin and with meningioma's attachment. Reconstruction of dura mater was conducted by a pedicled flap of own pericranium. Reconstruction of the bones was performed with the use of Codubix prosthesis (Tricomed, Poland). The patient was discharged in a good clinical condition, without neurological deficits, but receives anti-epileptic treatment in an outpatient clinic. MRI control examinations showed a complete removal of the meningioma (Fig. 3A, B).

Histopathologic study of tumour specimens showed WHO G II meningioma. In addition, the resected dura mater demonstrated immense inflammatory granulomatous reactions of the foreign body type, suspected to be related to the patient's allergy to, for instance, foreign agents. Thus, the skin allergic tests were performed in the outpatient clinic, using the "Prick" set by Allergopharma, which proved the patient's allergy to animal-origin proteins, saprophytes, mould and pollen of many plants. Moreover, ex post analysis of morphotic elements of peripheral blood revealed an increase in eosinophils, noted during and after the first operation; the results returned to normal after complete removal of the meningioma.

\section{Histopathological study}

The specimen from the $1^{\text {st }}$ operation consisted of tumour tissue in fragments of size 1.0 to $2.0 \mathrm{~cm}$. Histopathological examination of tumour demonstrated meningioma of grade WHO G I, with a mixed pattern of meningothelial, fibrous and angiomatous subtypes. In some parts of the tumour there were numerous blood vessels of various size, both thin-walled (Fig. 4A) or exhibiting greatly thickened, fibrous and hyalinized walls, often with prominent endothelial cells proliferation in intima (Fig. 4B). Some dispersed inflam- 

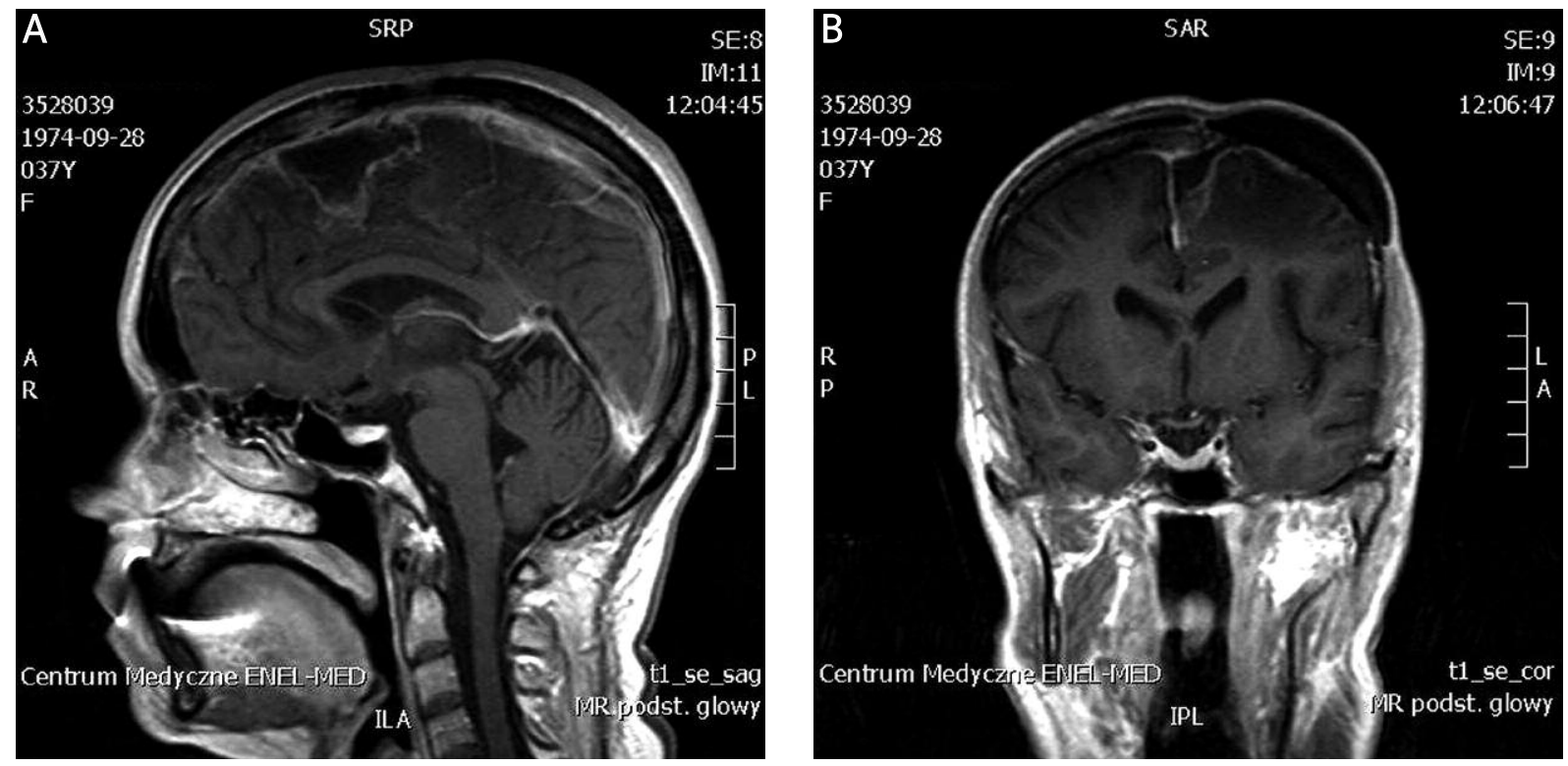

Fig. 3. MRI control examination after total tumour resection with contrast medium.

matory infiltrations of lymphocytic cells throughout the tumour were also observed. Rich collagen and reticulin fibers were seen in vascular and interstitial tissue. Mitoses were absent. The proliferative index of Ki-67 was low in tumour cells but focally increased in vascular walls.

The histopathological material from the second operation consisted of tumour tissue in fragments of size $1.5 \mathrm{~cm}$ to $2.5 \mathrm{~cm}$ and thickened dura mater in one fragment of size $4 \times 3 \times 0.5 \mathrm{~cm}$ and four fragments with longitudinal dimension of 1.0 to $4.5 \mathrm{~cm}$.

Microscopic examination of the tumour demonstrated meningioma, in parts with angiomatous and meningothelial appearance (Fig. 5A), and additionally, with chordoid areas characterized by cords of eosinophilic cells in a mucoid background (Fig. 5B). The tumour was classified as WHO G II meningioma because of the chordoid component. Mitoses were infrequent, a proliferative index of Ki-67 was about 5\%. Perivascular and interstitial infiltrations of lymphocytic and plasma cells were scattered throughout the tumour. In contrast, a microscopic study of dura mater revealed immense granulomatous necrotizing inflammation spreading from the internal surface of the dura matter (Fig. 6A). There were abundant infiltrations of lymphocytes, plasmocytes, scattered eosinophils and
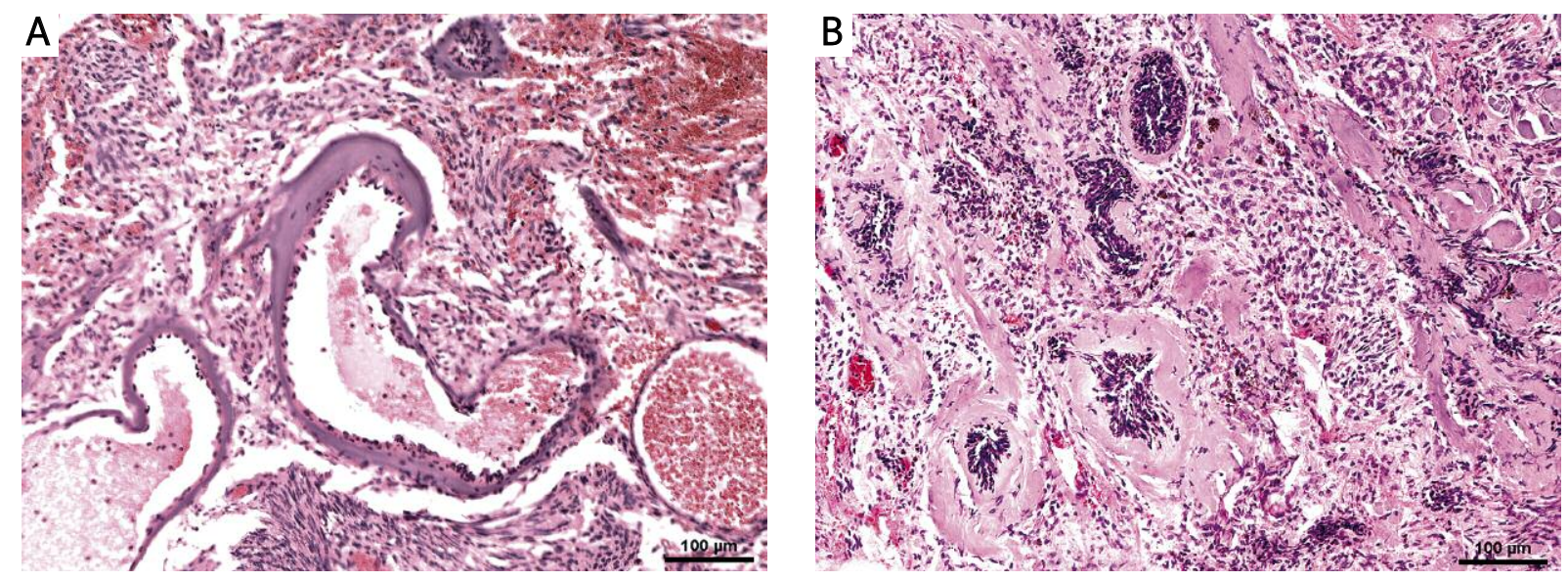

Fig. 4. Photomicrographs of meningioma after $1^{\text {st }}$ operation. A) Abnormal large thin- and thick-walled blood vessels within tumour. B) Numerous blood vessels with thicked and hyalinized walls and marked cellular proliferation of intima in some parts of meningioma. Staining with H\&E. 

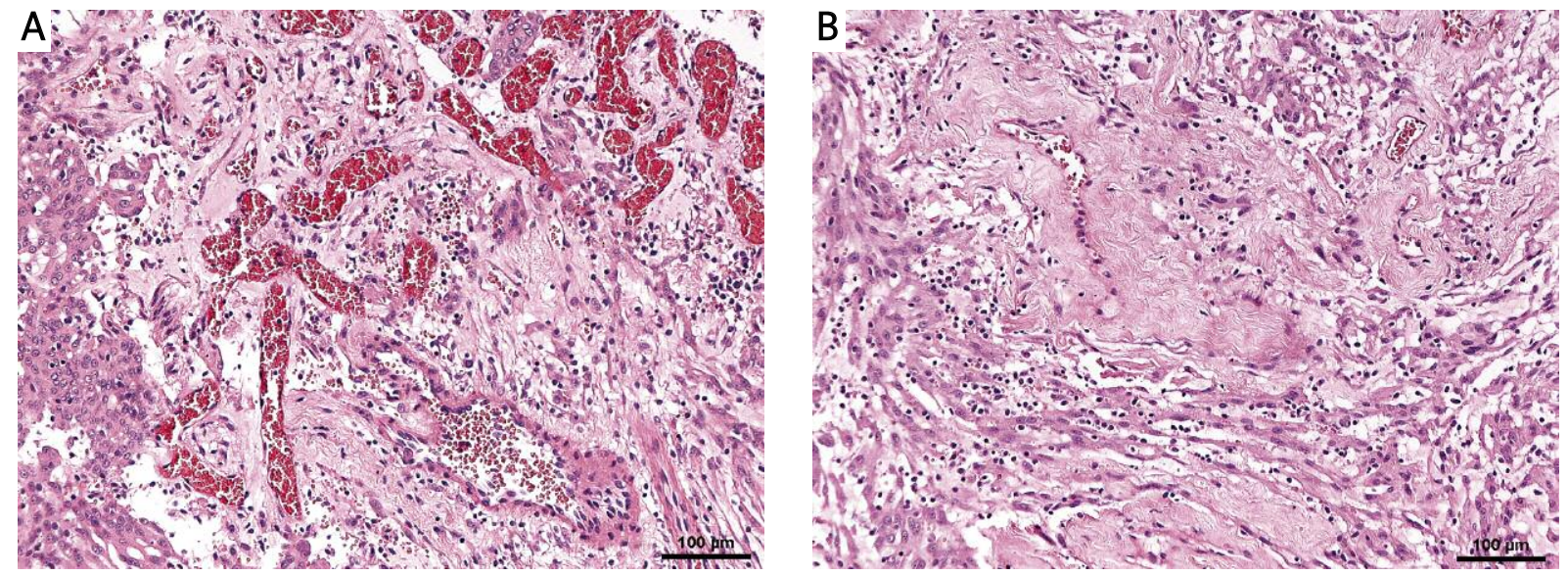

Fig. 5. Photomicrographs of meningioma after second operation. A) Part of meningioma with angiomatous appearance. B) Cords of meningioma cells in a mucoid background as well as several vessels rich in collagen and perivascular infiltration of lymphocytic cells in the tumour. Staining with H\&E.
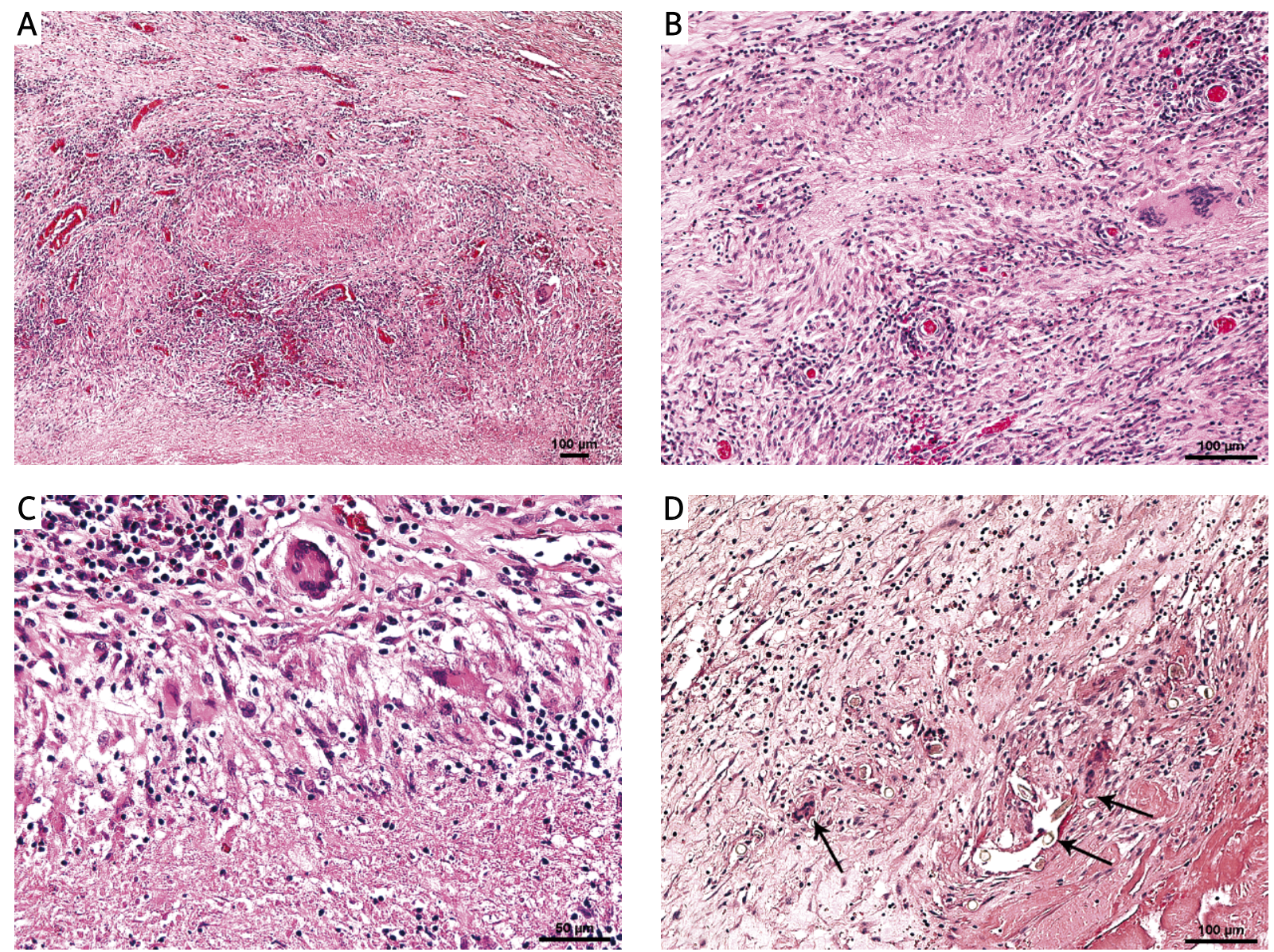

Fig. 6. Photomicrographs of dura matter after second operation. A) Diffuse dense inflammatory infiltrates with necroses in dura mater. B) Granulomatous necrotizing inflammation with multinucleated giant cells and lymphocytic et eosinophilic cell infiltrates. C) Granuloma with caseous necrosis surrounded by infiltration of epthelioid cells and Langhans type multinucleated giant cells. D) Scattered multinucleated cells of foreign body type (arrows) connecting small particles of foreign material, presumably Oxycel, within loose granulation tissue and slight lymphocyte infiltrations. Staining with H\&E (A-D). 

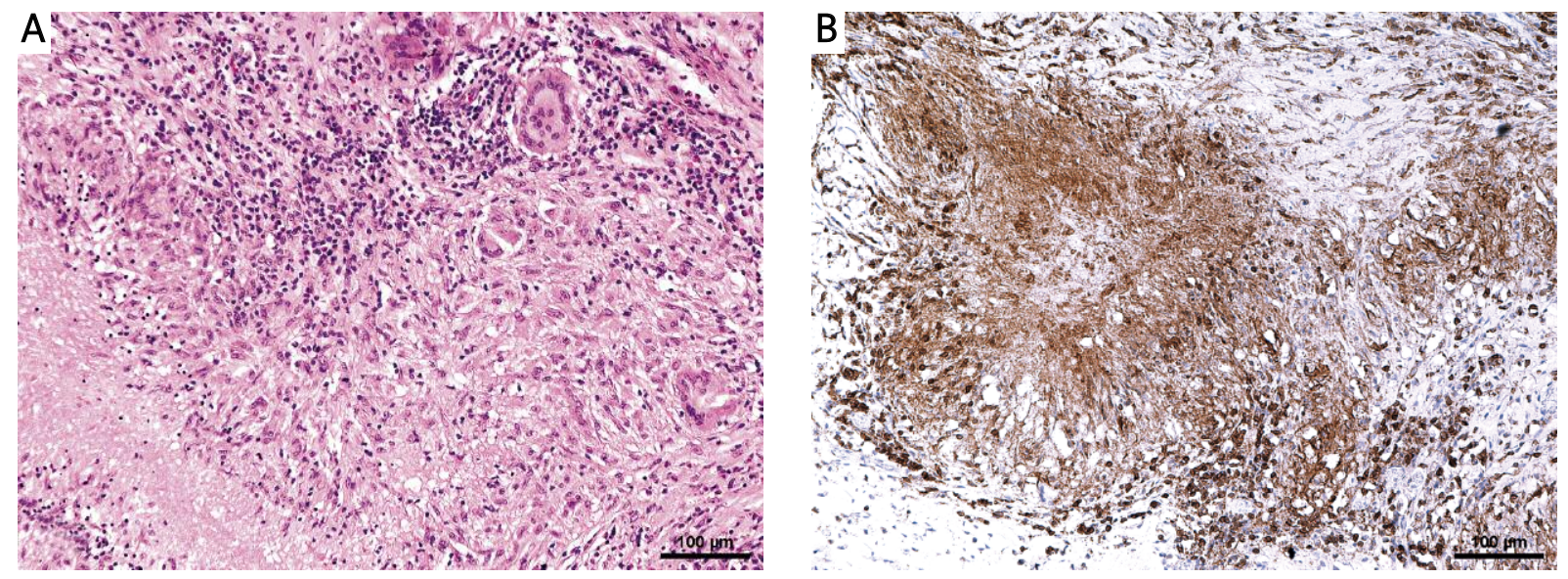

Fig. 7. A) Massive caseating granulomas with giant multinucleated cells, both of the Langhans and foreign body types. Staining with H\&E. B) Immunohistochemical staining for CD45 show strong cellular reaction in granuloma.

multinucleated giant cells (Fig. 6B). Many granuloma formations, exhibiting central caseous necrosis surrounded by epithelioid cells as well as giant multinucleated cells, both of the foreign body and Langhans type, were seen (Fig. 6B, C). Outside the major mass of inflammation, there were single multinucleated cells of the foreign body type, which were connected with a few small fragments of foreign material (Fig. 6D), primarily resembling degraded Oxycel (acc. to Ribalta et al. 2004) [14]. However, no particles of foreign material were identified within dense granulomatous infiltrations of dura matter (Fig. 7A). Staining for Mycobacteria with Ziehl-Neelsen method was negative. Immunohistochemically, the inflammatory cells in granulomas showed strong staining for CD 45 (Fig. 7B), whereas the cells immunopositive for CD20 were less frequent.

\section{Discussion}

Discrepancies concerning assessment of biopsy material of the same benign tumour after the first and second stage of surgical treatment was a subject of clinical discussion. A local inflammatory process in the specimen of tumour and granulomatous inflammation in the dura mater indicated a factor present in situ after the first stage of surgical treatment. Bacterial and infectious aetiology was excluded due to no local and no clinical symptoms, negative laboratory tests and correct healing of the wound. However, slight symptoms of skin allergy and face swelling reactions were observed by the patient after the first operation, similar to earlier allergic reactions to environmental allergens.
The fact that haemostatic and insulation materials used in the surgery may trigger local granulomatous reaction is known and documented but the application of these agents during surgical procedures is so common that this constitutes a kind of safety policy.

In the discussions, there exist opinions that autogenous pedicled flap is, undoubtedly, the best insulation, reconstructive, and covering material, without the risk of granuloma formation [5,6,17].

The goal of conducting and finishing the correct surgical procedure is effective haemostasis. Agents that locally aid haemostasis are exogenous in nature and during a neurosurgical procedure, they are applied directly on the bleeding surface and stay in the cerebral wound, giving the slow resorption. In neurosurgical practice the local granulomatous reaction after application of materials supporting haemostasis is not common. There is, however, an opinion that an excessive quantity of locally applied agents and their diversity may lead to intensified local reaction against the foreign body (foreign material) and thus formation of a granuloma [8]. This can also be caused by individual hypersensitivity of the patient to foreign proteins that might be an allergic factor.

In our case, local granulomatous infiltrations were observed in the resected dura mater specimens during the second stage of surgery of a huge meningioma. We have speculated that this inflammatory reaction was most likely due to the haemostatics used at the time of the first surgery. Evidence of small pieces of foreign body elements, mainly appearing as hollow "ghost" fibers of degraded Oxycel, was histologically found in proximity to granulomas. 
The two-stage procedure of treatment of a huge intracranial meningioma is accepted from the safety point of view. Assessment of intracranial meningioma resection is performed using the Simpson's scale [7]. A decision to finish the operation is determined by many factors, e.g. surgical experience, local and general situation, blood loss, anaesthetic indication etc. The effective haemostasis during surgery is the main point to decide about finish the procedure.

In our case during the operation, in order to support the local haemostasis of the meningioma's vessels, haemostatic flakes were used - Oxycel (oxidized cellulose) and Spongostan (gelatin sponge) obtained from swine gelatin. To cover the lack of dura mater, own pericranium was sewn in and additionally artificial material made from horse collagen and covered with human fibrinogen and human thrombin - TachoSil - was used.

After the first operation no clinical symptoms of infection were observed. The second surgical stage also ended successfully. No improper clinical course was observed during outpatient clinic assessment, therefore we concluded complete sterility of the intracranial processes taking place and being a reaction to the applied materials.

The literature contains examples of sterile formation of intracranial granuloma $[1,4,9]$. Undesirable effects in the form of granulomatous processes in the place where artificial insulating, sealant and haemostatic materials or stitches were used are described in the literature [4,9,12-14]. Cases of granulomas formation of various sizes and locations, causing clinical symptoms of a local mass effect or suggesting re-growth of the tumour were also published [11,14-16]. Attention is paid to the fact that application of too many artificial materials can increase the risk of the foreign body granuloma formation, i.e. after using Goretex, autologous powdered bone, bone cement and fibrin glue in order to reconstruct the damaged dura [8].

In most publications the diagnosis of intracranial granulomatous reaction resulted from the symptoms of the local mass effect, which were observed several months after the end of surgical treatment [3,8,9, $15,16]$. In our case the interval between two operations was 3 months and the mass effect caused by local granulomatous process was not clinically observed. The literature does not mention standard examinations (allergic test) related to allergies to animal proteins during planned operations of intracranial meningiomas. In our opinion, during planned reconstruction procedures special attention should be paid to persons with a case history related to possible allergies to animal and human proteins, in the context of applying various exogenous materials. There are reports suggesting allergic aetiology caused by heterogeneous collagen used in haemostasis, published after a childneurosurgical procedure [2]. One should also use a local preventive form of avoiding too large quantity of haemostatic agents and artificial insulation materials and, where possible, use an autogenous one. It is also significant to use new technologies, at the level of nanoparticles, in order to work out the best tolerated materials.

\section{References}

1. Aoki N, Sakai T, Oikawa A. Postoperative inflammatory reaction developing focal but severe brain edema. A possible complication of topical application of Biobond-soaked oxycellulose. Acta Neurol Scand 1998; 98: 288-291.

2. Apel-Sarid L, Cochrane DD, Steinbok P, Byrne AT, Dunham C. Microfibrillar collagen hemostat-induced necrotizing granulomatous inflammation developing after craniotomy: a pediatric case series. Neurosurg Pediatr 2010; 6: 385-392.

3. Feldman RP, Marcovici A, Suarez M, Goodrich JT. Foreign body granuloma mimicking intracranial meningioma: case report and review of the literature. Neurosurgery 1999; 44: 855-858.

4. Ganau M, Nicassio N, Tacconi L. Postoperative aseptic intracranial granuloma: the possible influence of fluid hemostatics. Case Rep Surg 2012; 614321. Epub 2012 Aug 9.

5. Goel A. Long vascular pedicle cranial flap. Br J Neurosurg 1995; 9 : 667-670.

6. Goel A. Multilayer reconstruction of the anterior cranial fossa floor. Br J Neurosurg 1998; 12: 254-258.

7. Halliday J, Fernandes H. Meningioma recurrence: the efficacy and cost-effectiveness of current screening. Br J Neurosurg 2010; 24 : 55-61.

8. Hata N, Hisada K, Torisu R, Suzuki SO, Kameda K, Sasaki T. Foreign body granuloma associated with dura -cranioplasty after resection of convexity meningioma with extracranial extension: case report. Neurol Med Chir (Tokyo) 2011; 51: 236-238.

9. Ito H, Onishi H, Shoin K, Nagatani H. Granuloma caused by oxidized cellulose following craniotomy. Acta Neurochir (Wien) 1989; 100: 70-73.

10. Klimo P (Jr), Khalil A, Slotkin JR, Smith ER, Scott RM, Goumnerova LC. Wound complications associated with the use of bovine serum albumin-glutaraldehyde surgical adhesive in pediatric patients. Neurosurgery 2007; 60 (Suppl 2): 305-309.

11. Kothbauer KF, Jallo GI, Siffert J, Jimenez E, Allen JC, Epstein FJ. Foreign body reaction to hemostatic materials mimicking recurrent brain tumor. Report of three cases. J Neurosurg 2001; 95: 503-506.

12. Korosue K, Tamaki N, Matsumoto S, Ohi Y. Intracranial granuloma as an unusual complication of subdural peritoneal shunt. Case report. J Neurosurg 1981; 55: 136-138. 
13. Onodera H, Furuya Y, Uchida M, Nakayama H, Nakamura H, Sakakibara Y, Taguchi Y. Intracranial foreign body granuloma caused by dural tenting suture. Br J Neurosurg 2011; 25: 652-654.

14. Ribalta T, McCutcheon IE, Neto AG, Gupta D, Kumar AJ, Biddle DA, Langford LA, Bruner JM, Leeds NE, Fuller GN. Textiloma (gossypiboma) mimicking recurrent intracranial tumor. Arch Pathol Lab Med 2004; 128: 749-758.

15. Saeidiborojeni HR, Fakheri T, lizadi B. Intracranial foreign body granuloma simulating brain tumor: a case report. J Res Med Sci 2011; 16: 358-360.

16. Sandhu GS, Elexpuru-Camiruaga JA, Buckley S. Oxidized cellulose (Surgicel) granulomata mimicking tumour recurrence. Br J Neurosurg 1996; 10: 617-619.

17. Scher RL, Cantrell RW. Anterior skull base reconstruction with the pericranial flap after craniofacial resection. Ear Nose Throat J 1992; 71: 210-212, 215-217. 